\title{
ON THE K-STABILITY OF FANO VARIETIES AND ANTICANONICAL DIVISORS
}

\author{
KENTO FUJITA, YUJI ODAKA
}

\begin{abstract}
We apply a recent theorem of Li and the first author to give some criteria for the K-stability of Fano varieties in terms of anticanonical $\mathbb{Q}$-divisors. First, we propose a condition in terms of certain anti-canonical $\mathbb{Q}$-divisors of given Fano variety, which we conjecture to be equivalent to the K-stability. We prove that it is at least sufficient condition and also relate to the Berman-Gibbs stability. We also give another algebraic proof of the K-stability of Fano varieties which satisfy Tian's alpha invariants condition.
\end{abstract}

\section{INTRODUCTION}

In this short paper, we discuss the K-stability of Fano varieties, which is an algebro-geometric stability condition originally motivated by studies of Kähler metrics. Indeed, as expected, when the base field is the complex number field, it is recently established that the existence of positive scalar curvature Kähler-Einstein metrics, i.e., Kähler metrics with constant Ricci curvature, is actually equivalent to the algebrogeometric condition "K-stability", by the works of [DT92, Tia97, Don05, CT08, Sto09, Mab08, Mab09, Ber16] and recent celebrated CDS15a, CDS15b, CDS15c, Tia15. This equivalence had been known before as the Yau-Tian-Donaldson conjecture (for the case of Fano varieties).

It also turned out that such canonical Kähler metrics and K-stability play crucial roles for nice moduli theory (cf., [FS90, Od10, DS14, Od13]), and indeed recently OSS16] constructed compact moduli spaces of smoothable Kähler-Einstein Fano varieties of two dimension and [LX14, SSY14, Od15] extended to higher dimensional case. The current approaches heavily depend on [DS14] and again the above-mentioned Yau-Tian-Donaldson equivalence.

2010 Mathematics Subject Classification. Primary 14J45; Secondary 14L24.

Key words and phrases. Fano varieties, K-stability, Kähler-Einstein metrics

K.F. is Partially supported by JSPS Fellowship for Young Scientists. Y.O. is Partially supported by JSPS Kakenhi no. 30700356 (Wakate (B)). 
However, it has been known as a difficult problem to test K-stability for given Fano varieties. Our purpose here is to develop algebraic studies of K-stability of Fano varieties one step further, mainly after [Li16, Fjt16b].

In this paper, we treat a stronger version of the K-stability introduced by Dervan Der14 and Boucksom-Hisamoto-Jonsson BHJ15] which is called uniform K-stability. The notion is expected to be eventually equivalent to the (original) K-stability.

We start with fixing our notation as follows.

\section{Notation.}

- We work over an arbitrary algebraic closed field of characteristic zero throughout the paper.

- $X$ is a $\mathbb{Q}$-Fano variety of dimension $n$, which means a log terminal projective variety with ample $\mathbb{Q}$-Cartier anticanonical divisor.

- (cf., KM98, Definition 2.24]) $F$ is a prime divisor over $X$, which means the equivalence class of an irreducible reduced Weil divisor on a normal blow up $Y$ of $X$ up to strict transform. In this paper, we occasionally denote the blow up by $\sigma: Y \rightarrow X$ . See [KM98, §2.3] for the details of the basic related materials.

- For $k \in \mathbb{Z}_{\geq 0}$ and $x \in \mathbb{R}_{\geq 0}$, let $H^{0}\left(-k K_{X}-x F\right)$ be the subspace of $H^{0}\left(-k \bar{K}_{X}\right)$ whose sections vanishing along the generic point of $F$ at least $x$ times.

- For $x \in \mathbb{R}_{\geq 0}$, we set

$$
\operatorname{vol}\left(-K_{X}-x F\right):=\limsup _{k \rightarrow \infty} \frac{h^{0}\left(-k K_{X}-k x F\right)}{k^{n} / n !} .
$$

- $A_{X}(F)$ denotes the $\log$ discrepancy of $X$ along $F$ and $\tau(F)$ denotes the pseudo-effective threshold of $-K_{X}$ with respect to $F$, i.e.,

$$
\tau(F):=\sup \left\{\frac{a}{k} \mid a, k \in \mathbb{Z}_{>0} \text { s.t., } H^{0}\left(-k K_{X}-a F\right) \neq 0\right\} .
$$

We also denote the log discrepancy of $(X, \Delta)$ along $F$ by $A_{(X, \Delta)}(F)$, where $\Delta$ is an $\mathbb{R}$-divisor on $X$ with $K_{X}+\Delta \mathbb{R}$-Cartier.

- For an effective $\mathbb{R}$-Cartier divisor $D$ on $X$,

$$
\operatorname{lct}(X ; D):=\max \left\{c \in \mathbb{R}_{\geq 0} \mid(X, c D): \log \text { canonical }\right\}
$$

be the log canonical threshold of $X$ along $D$.

A key notion we introduce is the following special type of anticanonical $\mathbb{Q}$-divisors. 
Definition 0.1. Let $k$ be a positive integer. Given any basis

$$
s_{1}, \ldots, s_{h^{0}\left(-k K_{X}\right)}
$$

of $H^{0}\left(-k K_{X}\right)$, taking the corresponding divisors $D_{1}, \ldots, D_{h^{0}\left(-k K_{X}\right)}$ $\left(D_{i} \sim-k K_{X}\right)$, we get an anticanonical $\mathbb{Q}$-divisor

$$
D:=\frac{D_{1}+\cdots+D_{h^{0}\left(-k K_{X}\right)} .}{k \cdot h^{0}\left(-k K_{X}\right)} .
$$

We call this kind of anticanonical $\mathbb{Q}$-divisor an anticanonical $(\mathbb{Q}$ - $)$ divisor of $k$-basis type.

Definition 0.2. For $k \in \mathbb{Z}_{>0}$, set

$$
\delta_{k}(X):=\inf _{\substack{\left(-K_{X} \sim_{\mathbb{Q}}\right) D ; \\ D: k \text {-basis type }}} \operatorname{lct}(X ; D) .
$$

Moreover, we define

$$
\delta(X):=\limsup _{k \rightarrow \infty} \delta_{k}(X)
$$

Then we prove the following criterion.

TheOREM 0.3 (K-stability criteria via basis type divisors). Let $X$ be $a \mathbb{Q}$-Fano variety. If $\delta(X)>1$ (resp., $\geq 1$ ) then $\left(X,-K_{X}\right)$ is uniformly K-stable (resp., K-semistable).

Reviewing our proof of Theorem 2.1, we expect that the converse is also true though some technical difficulties is preventing us from proving it at the moment.

Conjecture 0.4 (Main conjecture). Let $X$ be a $\mathbb{Q}$-Fano variety. Then the K-stability (resp., K-semistability) of $\left(X,-K_{X}\right)$ is equivalent to $\delta(X)>1$ (resp., $\delta(X) \geq 1)$.

From Theorem 0.3 , we get that the Berman-Gibbs stability (resp., semistability) of $X$ implies K-stability (resp., K-semistability). The Berman-Gibbs stability was introduced in Berman [Ber13] (see Definition 2.4 for the definition) and the following algebraic result was known by Fjt16a (cf. Ber13, §7]). However, we should emphasize that the proof of Theorem 0.5 is much easier than the proof of [Fjt16a].

It also follows from our arguments that if the Berman-Gibbs stability is actually equivalent to the K-stability, it also implies our equivalence conjecture 0.4 .

TheOrem 0.5 (cf. Ber13, Fjt16a). Let $X$ be a $\mathbb{Q}$-Fano variety. If $X$ is Berman-Gibbs stable (resp., Berman-Gibbs semistable), then $X$ is uniformly K-stable (resp., K-semistable). (For the definition of Berman-Gibbs stability, see Section 2.2.) 
Finally, we also give a new algebraic (re-)proof of the following Tian's famous criterion via the alpha invariant [Tia87] (see Theorem 3.2 in detail). The first algebraic proof of it is by the second author and Sano OS12 but our argument here is very different. Indeed, we work on $X$ itself and its valuations, thus in $n$-dimensional geometry, while the proof of OS12] was via analysis of test configurations, thus essentially depends on $(n+1)$-dimensional geometry.

TheOREM 0.6 (cf., TTia87, OS12, Der14, BHJ15]). For an $n$ dimensional $\mathbb{Q}$-Fano variety $X$, if $\alpha(X)>($ resp., $\geq) n /(n+1)$, then $\left(X,-K_{X}\right)$ is uniformly $K$-stable (resp., K-semistable).

In this paper, we omit the original definition of the K-stability and for that, simply refer to the original [Tia97], [Don02], and for uniform version, to [Der14], BHJ15]. The reason is that, as in the next section (Theorem 1.1) we start from review of the results of [Fjt16b], [Li16] which can be seen as giving an alternative definition of the (uniform) K-(semi)stability of Fano varieties. Therefore, we do not need the original definition logically in this paper. After the review as Theorem 1.1. we also slightly modify the uniform K-stability part of it to the form we use in the following sections. In section 2, we prove Theorem 0.3, the criterion via basis type divisor (in the sense of 0.1 ) and discuss relation with the Berman-Gibbs stability [Ber13. In the last section, we discuss the relation with the alpha invariant.

Acknowledgement. We are grateful for Yuchen Liu who let the first author knows that he independently found Theorem 3.5.

\section{VAluatiVE CRITERIA OF K-STABILITY AND THEIR VARIANT}

In this section, as a preparation, we recall the key theorem by Li16, Fjt16b and also prove a slight variant which we use in this paper.

TheOREm 1.1. Let $X$ be a $n$-dimensional $\mathbb{Q}$-Fano variety. For an arbitrary prime divisor $F$ over $X$, we set

$$
\begin{aligned}
\beta(F) & :=A_{X}(F)\left(-K_{X}\right)^{\cdot n}-\int_{x=0}^{\infty} \operatorname{vol}\left(-K_{X}-x F\right) d x, \\
j(F) & :=\int_{x=0}^{\tau(F)}\left(\left(-K_{X}\right)^{\cdot n}-\operatorname{vol}\left(-K_{X}-x F\right)\right) d x .
\end{aligned}
$$

(When $F$ is a bona fide divisor on $X, \beta(F)$ first appeared in [Fjt15a.) Then we have

(i) $\left([\right.$ Li16, Fjt16b $)\left(X,-K_{X}\right)$ is K-semistable if and only if $\beta(F) \geq$ 0 for any $F$. Moreover, the pair is K-stable if $\beta(F)>0$ for any $F$. 
(ii) $($ Fjt16b $)\left(X,-K_{X}\right)$ is uniformly K-stable if and only if there exists a positive real number $\delta$ such that $\beta(F) \geq \delta \cdot j(F)$ for any $F$.

The uniform K-stability treated in (iii) above is introduced by Dervan [Der14, Boucksom-Hisamoto-Jonsson [BHJ15] as a conjecturally equivalent variant of the K-stability. [Der14] refers to it as K-stability with respect to the minimum norm and [BHJ15] refers to it as J-uniform K-stability or simply the uniform K-stability. In this paper, as there should be no confusion, we simply call it as the uniform K-stability. We also note that the above conditions for K-stability resembles the definition of $\log$ terminality, log canonicity.

In this section, we prepare a variant of the above Theorem 1.1 (iii). Here, we prepare the following simple lemma.

\section{LEMMA 1.2 .}

$$
\left(-K_{X}\right)^{\cdot n} \tau(F) \geq \int_{x=0}^{\infty} \operatorname{vol}\left(-K_{X}-x F\right) d x \geq \frac{1}{n+1}\left(-K_{X}\right)^{\cdot n} \tau(F) .
$$

Proof. The first inequality follows straightforward from $\operatorname{vol}\left(-K_{X}-\right.$ $x F) \leq\left(-K_{X}\right)^{\cdot n}$. The second inequality follows from the result of the concavity of volume function (cf., e.g., [LM09]); we can see the inequality $\operatorname{vol}\left(-K_{X}-x F\right) \geq\left(-K_{X}\right)^{\cdot n}\left(\frac{x}{\tau(F)}\right)^{n}$.

This Lemma 1.2 allows us to give a version of the above Theorem 1.1 (iii), i.e., the uniform stability criterion in Fjt16b.

THEOREM 1.3. Suppose that a $\mathbb{Q}$-Fano variety $X$ satisfies that there is a positive real constant $\varepsilon>0$ such that for any prime divisor $F$ over $X$, we have

$$
(1-\varepsilon) A_{X}(F)\left(-K_{X}\right)^{\cdot n} \geq \int_{x=0}^{\infty} \operatorname{vol}\left(-K_{X}-x F\right) d x .
$$

Then $\left(X,-K_{X}\right)$ is uniformly K-stable.

Proof. The assumption can be rewritten as, by Lemma 1.2, that there exists a positive real number $\varepsilon^{\prime}$ satisfying

$$
A_{X}(F)\left(-K_{X}\right)^{\cdot n} \geq \int_{x=0}^{\infty} \operatorname{vol}\left(-K_{X}-x F\right) d x+\varepsilon^{\prime} \tau(F)\left(-K_{X}\right)^{\cdot n},
$$

for any divisor $F$ over $X$. On the other hand, the desired inequality $\beta(F)>\delta \cdot j(F)$ of Theorem 1.1 (iii) can be straightforwardly re-written as

$$
\left(\left(1+\delta^{\prime}\right) A_{X}(F)-\delta^{\prime} \tau(F)\right)\left(-K_{X}\right)^{\cdot n} \geq \int_{x=0}^{\infty} \operatorname{vol}\left(-K_{X}-x F\right) d x
$$


by putting $\delta^{\prime}:=\delta /(1-\delta)$. The inequality (1) obviously implies (2) when $\delta^{\prime}=\varepsilon^{\prime}$ as $\delta^{\prime} A_{X}(F)>0$ from the $\log$ terminality assumption of $X$.

\section{Via "BASIS TYPE" ANTICANONICAL DIVISORS}

2.1. K-stability and basis type divisors. Our main theorem in this section is as follows.

TheOREM 2.1 (K-stability criteria via basis type divisors). Let $X$ be $a \mathbb{Q}$-Fano variety. Let $\delta(X)$ be the value in Definition 0.2. If $\delta(X)>1$ $($ resp., $\geq 1)$ then $\left(X,-K_{X}\right)$ is uniformly K-stable (resp., K-semistable).

We call the above conditions asymptotic klt (resp., asymptotic log canonicity of the (set of) log Calabi-Yau pairs $\{(X, D)\}$.

Proof of Theorem 2.1. Take any prime divisor $F$ over $X$. First we explain the key observation as the following lemma, which describes the minimum of $\log$ discrepancies of the log pairs associated to $k$-basis type divisors.

Lemma 2.2 (Log discrepancy formula). For $c>0$, we have

$$
\min _{D: k \text {-basis type }} A_{(X, c D)}(F)=A_{X}(F)-c \frac{\sum_{1 \leq a} h^{0}\left(-k K_{X}-a F\right)}{k \cdot h^{0}\left(-k K_{X}\right)} .
$$

In particular, the left hand side (the minimum) exists.

Proof of Lemma 2.2. Take any basis $s_{1}, \ldots, s_{h^{0}\left(-k K_{X}\right)}$ of $H^{0}\left(-k K_{X}\right)$ and associate the $k$-basis type divisor $D$. Changing the order if necessary, we can and do assume that there is a decreasing sequence $h^{0}\left(-k K_{X}\right) \geq i_{1} \geq \cdots \geq i_{k} \geq \cdots \geq 0$ such that the valuations along $F$ are $v_{F}\left(s_{j}\right)=a$ if $i_{a} \geq j>i_{a+1}$. Then

$$
v_{F}(D)=\frac{\sum_{0 \leq a} a\left(i_{a}-i_{a+1}\right)}{k \cdot h^{0}\left(-k K_{X}\right)}=\frac{\sum_{1 \leq a} i_{a}}{k \cdot h^{0}\left(-k K_{X}\right)}
$$

for the corresponding $k$-basis type anticanonical $\mathbb{Q}$-divisor $D$. Here, $v_{F}$ denotes the valuation along $F$. Linear independence of $\left\{s_{i}\right\}_{i}$ implies $i_{a} \leq h^{0}\left(-k K_{X}-a F\right)$ whose equality holds for when the basis is compatible with the filtration $\left\{H^{0}\left(-k K_{X}-a F\right)\right\}_{a \geq 0}$. Hence,

$$
\sup _{D: k \text {-basis type }} v_{F}(D)=\frac{\sum_{1 \leq a} h^{0}\left(-k K_{X}-a F\right)}{k \cdot h^{0}\left(-k K_{X}\right)} .
$$


The definition of $\delta_{k}(X)$ implies that

$$
A_{X}(F)-\delta_{k}(X) \frac{\sum_{1 \leq a} h^{0}\left(-k K_{X}-a F\right)}{k \cdot h^{0}\left(-k K_{X}\right)} \geq 0
$$

by Lemma 2.2. Note that

$$
\lim _{k \rightarrow \infty} \frac{\sum_{1 \leq a} h^{0}\left(-k K_{X}-a F\right)}{k \cdot h^{0}\left(-k K_{X}\right)}=\frac{\int_{0}^{\tau(F)} \operatorname{vol}\left(-K_{X}-x F\right) d x}{\left(-K_{X}\right)^{\cdot n}} .
$$

Thus the assertion follows from Theorem 1.3 .

REMARK 2.3. It is not true that $(X, D)$ is always log canonical for any basis type anticanoncal $\mathbb{Q}$-divisor on any K-semistable Fano variety $X$. Indeed, we checked by Macaulay2 that the Ono-Sano-Yotsutani's example OSY12] does not satisfy the condition.

2.2. Relation with the Berman-Gibbs stability. In this subsection, we discuss relations of our approach to the Berman-Gibbs stability introduced by Berman Ber13. It is also defined in terms of (pluri)anticanonical divisors but that of large self product of the given Fano variety. First, let us recall the notion.

Definition 2.4 (see [Ber13] and Fjt16a]). Let $X$ be a $\mathbb{Q}$-Fano variety. Consider any $k \in \mathbb{Z}_{>0}$ with $-k K_{X}$ globally generated Cartier. We set

- $N_{k}:=h^{0}\left(-k K_{X}\right)$,

- $\phi_{k}:=\phi_{\left|-k K_{X}\right|}: X \rightarrow \mathbb{P}^{N_{k}-1}$,

- $\Phi_{k}:=\phi_{k} \times \cdots \times \phi_{k}: X^{N_{k}} \rightarrow\left(\mathbb{P}^{N_{k}-1}\right)^{N_{k}}$,

- $\mathcal{D}_{k}:=\Phi_{k}^{*} \operatorname{Det}_{N_{k}}$, where $\operatorname{Det}_{N_{k}} \subset\left(\mathbb{P}^{N_{k}-1}\right)^{N_{k}}$ is the determinantal divisor,

$$
\gamma_{k}(X):=\operatorname{lct}_{\Delta_{X}}\left(X^{N_{k}} ; \frac{1}{k} \mathcal{D}_{k}\right)
$$

where $\Delta_{X} \subset X^{N_{k}}$ is the diagonal.

Moreover, we define

$$
\gamma(X):=\liminf _{\substack{k \rightarrow \infty \\-k K_{X}: \text { Cartier }}} \gamma_{k}(X)
$$

The $X$ is said to be Berman-Gibbs stable (resp. Berman-Gibbs semistable) if $\gamma(X)>1$ (resp. $\gamma(X) \geq 1$ ) holds.

The main purpose of this section is to give another simpler proof of Fjt16a, Theorem 1.4] from our perspective. 
TheOREM 2.5. Let $X$ be a $\mathbb{Q}$-Fano variety. For any $k \in \mathbb{Z}_{>0}$ with $-k K_{X}$ Cartier, we have the inequality $\delta_{k}(X) \geq \gamma_{k}(X)$. In particular, we get the inequality $\delta(X) \geq \gamma(X)$.

Together with Theorem 2.1, this immediately implies the following:

Corollary 2.6 (cf. [Ber13, §7] and [Fjt16a, Theorem 1.4]). If $X$ is Berman-Gibbs stable (resp. Berman-Gibbs semistable), then $\left(X,-K_{X}\right)$ is uniformly K-stable (resp. K-semistable).

Proof of Theorem 2.5. Take any prime divisor $F$ over $X$ and take any $\log$ resolution $\sigma: Y \rightarrow X$ with $F$ smooth divisor on $Y$. For any $k \in$ $\mathbb{Z}_{>0}$ with $-k K_{X}$ Cartier, set $\psi_{k}:=\phi_{k} \circ \sigma: Y \rightarrow \mathbb{P}^{N_{k}-1}$. Then $\psi_{k}=$ $\phi_{\left|\sigma^{*}\left(-k K_{X}\right)\right|}$, and $\Psi_{k}:=\left(\phi_{k} \circ \sigma\right)^{N_{k}}=\Phi_{k} \circ \sigma^{N_{k}}: Y^{N_{k}} \rightarrow\left(\mathbb{P}^{N_{k}-1}\right)^{N_{k}}$ satisfies that $\Psi_{k}^{*} \operatorname{Det}_{N_{k}}=\left(\sigma^{N_{k}}\right)^{*} \mathcal{D}_{k}$. Thus the multiplicity of $\left(\sigma^{N_{k}}\right)^{*} \mathcal{D}_{k}$ along $F^{N_{k}}=F \times \cdots \times F \subset Y^{N_{k}}$ is bigger than or equal to

$$
\sum_{j=1}^{\infty} h^{0}\left(-k K_{X}-j F\right)
$$

On the other hand, we have

$$
\left(\sigma^{N_{k}}\right)^{*} K_{X^{N_{k}}}=K_{Y^{N_{k}}}-\sum_{\substack{F_{l} \subset Y \\ \sigma \text {-exceptional }}} \sum_{i=1}^{N_{k}}\left(A_{X}\left(F_{l}\right)-1\right) p_{i}^{*} F_{l}
$$

where $p_{i}: X^{N_{k}} \rightarrow X$ is the $i$-th projection morphism. Let $\mathcal{Z} \rightarrow Y^{N_{k}}$ be the blowup along $F^{N_{k}}$ and let $G \subset \mathcal{Z}$ be the exceptional divisor. Then we have

$0 \leq A_{\left(X^{\left.N_{k}, \frac{\gamma_{k}(X)}{k} \mathcal{D}_{k}\right)}\right.}(G) \leq N_{k} \cdot A_{X}(F)-\frac{\gamma_{k}(X)}{k} \sum_{j=1}^{\infty} h^{0}\left(-k K_{X}-j F\right)$.

This implies that

$$
A_{X}(F) \geq \frac{\gamma_{k}(X)}{k \cdot N_{k}} \sum_{j=1}^{\infty} h^{0}\left(-k K_{X}-j F\right) .
$$

Thus $A_{\left(X, \gamma_{k}(X) D\right)}(F) \geq 0$ for any $k$-basis type divisor $D$ and for any prime divisor $F$ over $X$ by Lemma 2.2. Hence we get the inequality $\delta_{k}(X) \geq \gamma_{k}(X)$.

Remark 2.7. From Theorem 2.5, it also follows that if the BermanGibbs stability is equivalent to the K-stability (cf., [Ber13, §7]), our Conjecture 0.4 is also true. 


\section{RELATiON With THE ALPha INVARIANT}

Let us recall the basic of the alpha invariants [Tia87] which was first introduced in terms of the Kähler potentials. Later it was proved to be the same as the following algebraic version global log canonical threshold which we use.

Theorem 3.1 (cf., Dem08]). For an arbitrary Fano manifold $X$, $\alpha(X)=\sup \left\{\alpha>0 \mid(X, \alpha D)\right.$ is log canonical for any effective $\left.D \sim_{\mathbb{Q}}-K_{X}\right\}$.

In this paper, we treat the right hand side as the definition of alpha invariant as we do not think there would be some confusion. In particular, the definition naturally extends to $\mathbb{Q}$-Fano varieties.

The purpose of this section is to give an algebraic new proof of the following theorem from our perspective.

TheOrem 3.2 (cf., [Tia87, OS12, Der14, BHJ15]). For a $\mathbb{Q}-$ Fano variety $X$ of dimension $n$, if $\alpha(X)>($ resp., $\geq) n /(n+1)$, then $\left(X,-K_{X}\right)$ is uniformly K-stable (resp., K-semistable).

Given the equivalence 3.1 above, the above result can also be seen as a K-stability criterion in terms of anticanonical $\mathbb{Q}$-divisors. The statement is an algebraic counterpart of Tian's original statement that "For a Fano manifold $X$ with $\alpha(X)>n /(n+1)$, there exists a KählerEinstein metric" Tia87]. First algebraic proof of the K-stability (Theorem 3.2) was obtained by the first author and Sano [OS12] and then was later refined to the uniform K-stability by Dervan [Der14] and Boucksom-Hisamoto-Jonsson [BHJ15]. Both analyze the test configurations, thus the arguments are based on $(n+1)$-dimensional birational geometry. Our proof here is very different, in particular, is based on $n$ dimensional birational geometry and make use of the Okounkov body.

We prepare the following lemma in order to prove Theorem 3.2 .

Lemma 3.3. For any $\mathbb{Q}$-Fano variety $X$ and for any prime divisor $F$ over $X$, we have $\alpha(X) \cdot \tau(F) \leq A_{X}(F)$.

Proof. Assume that $k \in \mathbb{Z}_{>0}$ and $\tau \in \mathbb{R}_{>0}$ satisfies that $H^{0}\left(X,-k K_{X}-\right.$ $k \tau F) \neq 0$. Then we can find an effective $\mathbb{Q}$-divisor $D$ with $D \sim_{\mathbb{Q}}-K_{X}$ such that $A_{\left(X,\left(A_{X}(F) / \tau\right) D\right)}(F) \leq 0$. Thus we have $A_{X}(F) / \tau \geq \alpha(X)$.

Proof of Theorem 3.2. Assume that $\alpha(X)>n /(n+1)$ (resp. $\geq n /(n+$ $1))$. We can take $\delta \in(0,1)$ (resp. $\delta \in[0,1))$ such that $\delta \leq(n+$ $1)(\alpha(X)-(n /(n+1)))$. Pick any dreamy prime divisor $F$ over $X$ in the sense of Fjt16b, that is, the graded algebra

$$
\bigoplus_{k, j \in \mathbb{Z}_{\geq 0}} H^{0}\left(-k K_{X}-j F\right)
$$


is finitely generated.

Claim 3.4. There exists a normal projective variety $X^{\prime}$ with $-K_{X^{\prime}}$ $\mathbb{Q}$-Cartier and a prime divisor $F^{\prime}$ on $X^{\prime}$ which is $\mathbb{Q}$-Cartier such that:

- for any $k \in \mathbb{Z}_{>0}$ and $x \in \mathbb{R}_{>0}$ with $-k K_{X}$ Cartier, we have the equality

$$
H^{0}\left(-k K_{X}-x F\right)=H^{0}\left(X^{\prime}, k\left(-K_{X^{\prime}}+\left(A_{X}(F)-1\right) F^{\prime}\right)-x F^{\prime}\right),
$$

- for any $0<\varepsilon \ll 1,\left(-K_{X^{\prime}}+\left(A_{X}(F)-1\right) F^{\prime}\right)-\varepsilon F^{\prime}$ is ample.

Proof of Claim 3.4. Take any projective birational morphism $\sigma: Y \rightarrow$ $X$ with $Y$ smooth and $F \subset Y$. By [KKL12, Theorem 4.2] and [Fjt16b, Claim 6.3], we can find a birational contraction map $\varphi: Y \rightarrow X^{\prime}$ such that the strict transform $F^{\prime} \subset X^{\prime}$ of $F$ satisfies that the map $\varphi$ is the ample model of $\left(-K_{X^{\prime}}+\left(A_{X}(F)-1\right) F^{\prime}\right)-\varepsilon F^{\prime}$ for any $0<\varepsilon \ll 1$. (Indeed, the strict transform of $\sigma^{*}\left(-K_{X}\right)$ on $X^{\prime}$ is equal to $-K_{X^{\prime}}+$ $\left(A_{X}(F)-1\right) F^{\prime}$.) The divisor $\sigma^{*}\left(-K_{X}\right)$ is of course $\varphi$-nonnegative, and the divisor $\sigma^{*}\left(-K_{X}\right)-\varepsilon F$ is $\varphi$-nonpositive for any $0<\varepsilon \ll 1$. Thus the divisor $\sigma^{*}\left(-K_{X}\right)-x F$ is $\varphi$-nonpositive for any $x \in \mathbb{R}_{>0}$. Thus the assertion follows from [KKL12, Remark 2.4 (i)].

Set $H^{\prime}:=-K_{X^{\prime}}+\left(A_{X}(F)-1\right) F^{\prime}$. Fix any rational number $0<\varepsilon \ll$ 1. Take any complete flag in $X^{\prime}$ (in the sense of [LM09])

$$
X^{\prime} \supset Z_{1} \supset Z_{2} \supset \cdots \supset Z_{n}=\{\text { point }\}
$$

with $Z_{1}=F^{\prime}$. Let us consider the Okounkov body $\Delta_{\varepsilon}:=\Delta_{Z_{\bullet}}\left(H^{\prime}-\right.$ $\left.\varepsilon F^{\prime}\right) \subset \mathbb{R}_{\geq 0}^{n}$ of $H^{\prime}-\varepsilon F^{\prime}$ with respects to the flag $Z_{\bullet}$ (see [LM09] for the definition of the Okounkov bodies). Since $H^{\prime}-\varepsilon F^{\prime}$ is ample, by [LM09, Corollary 4.25] and Claim [3.4, we have

$$
\operatorname{vol}\left(\left.\Delta_{\varepsilon}\right|_{\nu_{1} \geq x-\varepsilon}\right)=\frac{1}{n !} \operatorname{vol}_{X^{\prime}}\left(H^{\prime}-x F^{\prime}\right)=\frac{1}{n !} \operatorname{vol}\left(-K_{X}-x F\right)
$$

for any $x \geq \varepsilon$, where

$$
\left.\Delta_{\varepsilon}\right|_{\nu_{1} \geq x-\varepsilon}:=\left\{\left(\nu_{1}, \ldots, \nu_{n}\right) \in \Delta_{\varepsilon} \mid \nu_{1} \geq x-\varepsilon\right\} .
$$

For any $x \geq \varepsilon$, let $Q(x)$ be the restricted volume of

$$
\left\{\left(\nu_{1}, \ldots, \nu_{n}\right) \in \Delta_{\varepsilon} \mid \nu_{1}=x-\varepsilon\right\} .
$$

Then we have

$$
\begin{aligned}
& \int_{\varepsilon}^{\tau(F)} \operatorname{vol}\left(-K_{X}-x F\right) d x=\int_{\varepsilon}^{\tau(F)} \int_{x}^{\tau(F)} n ! \cdot Q(y) d y d x \\
= & \int_{\varepsilon}^{\tau(F)} \int_{\varepsilon}^{y} n ! \cdot Q(y) d x d y=\int_{\varepsilon}^{\tau(F)} n ! \cdot(y-\varepsilon) Q(y) d y .
\end{aligned}
$$


Thus we get

$$
\frac{\int_{\varepsilon}^{\tau(F)} \operatorname{vol}\left(-K_{X}-x F\right) d x}{\operatorname{vol}\left(-K_{X}-\varepsilon F\right)}=\frac{\int_{\varepsilon}^{\tau(F)}(y-\varepsilon) Q(y) d y}{\int_{\varepsilon}^{\tau(F)} Q(y) d y} .
$$

The right-hand side is nothing but the first coordinate (say, $b_{1}$ ) of the barycenter of $\Delta_{\varepsilon}$. The value $b_{1}$ satisfies that $b_{1} \leq(\tau(F)-\varepsilon) \cdot n /(n+1)$ (see for example [Ham51]). This implies that

$$
\int_{0}^{\tau(F)} \operatorname{vol}\left(-K_{X}-x F\right) d x \leq \frac{n}{n+1} \tau(F) \operatorname{vol}\left(-K_{X}\right) .
$$

Thus the assertion follows from Lemma 3.3 and Theorem 1.3 (or, by Fjt16b, Theorem 1.3]).

We end our notes by observing the following lower bound of alpha invariant which is somewhat easier.

TheOrem 3.5. If a $\mathbb{Q}$-Fano variety $X$ of dimension $n$ is $K$-semistable, then $\alpha(X) \geq 1 /(n+1)$ holds.

Proof of Theorem 3.5. Take any $k \in \mathbb{Z}_{>0}$ with $-k K_{X}$ Cartier and take any $D \in\left|-k K_{X}\right|$. Then, by [Fjt15b, Theorem 4.10], we have

$$
\operatorname{lct}(X ; D) \cdot\left(-K_{X}\right)^{\cdot n} \geq \int_{0}^{\infty} \operatorname{vol}\left(-K_{X}-x D\right) d x .
$$

Since $\operatorname{vol}\left(-K_{X}-x D\right)=(1-k x)^{n}\left(-K_{X}\right)^{\cdot n}$ (if $\left.0 \leq x \leq 1 / k\right)$, we have $\operatorname{lct}(X ; D) \geq 1 /(k(n+1))$.

REMARK 3.6. Yuchen Liu independently obtained Theorem 3.5([Liu]).

\section{REFERENCES}

[Ber13] R. Berman, Kähler-Einstein metrics, canonical random point processes and birational geometry, arXiv:1307.3634.

[Ber16] R. Berman, K-polystability of Q-Fano varieties admitting Kähler-Einstein metrics, Invent. Math. 203 (2016), no. 3, 973-1025.

[BHJ15] S. Boucksom, T. Hisamoto and M. Jonsson, Uniform K-stability, Duistermaat-Heckman measures and singularities of pairs, arXiv:1504.06568.

[CDS15a] X. Chen, S. Donaldson and S. Sun, Kähler-Einstein metrics on Fano manifolds, I: approximation of metrics with cone singularities, J. Amer. Math. Soc. 28 (2015), no. 1, 183-197.

[CDS15b] X. Chen, S. Donaldson and S. Sun, Kähler-Einstein metrics on Fano manifolds, II: limits with cone angle less than $2 \pi$, J. Amer. Math. Soc. 28 (2015), no. 1, 199-234.

[CDS15c] X. Chen, S. Donaldson and S. Sun, Kähler-Einstein metrics on Fano manifolds, III: limits as cone angle approaches $2 \pi$ and completion of the main proof, J. Amer. Math. Soc. 28 (2015), no. 1, 235-278. 
[CT08] X. Chen and G. Tian, Geometry of Kähler metrics and foliations by holomorphic discs, Publ. Math. Inst. Hautes Études Sci. 107 (2008), 1-107.

[Dem08] J.-P. Demailly, Appendix to I. Cheltsov and C. Shramov's article. "Log canonical thresholds of smooth Fano threefolds : On Tian's invariant and log canonical thresholds, Russian Math. Surveys 63 (2008), no. 5, 945-950.

[Der14] R. Dervan, Uniform stability of twisted constant scalar curvature Kähler metrics, arXiv:1412.0648, to appear in Int. Math. Res. Not. IMRN.

[Don02] S. Donaldson, Scalar curvature and stability of toric varieties, J. Differential Geom. 62 (2002), no. 2, 289-349.

[Don05] S. Donaldson, Lower bounds on the Calabi functional, J. Differential Geom. 70 (2005), no. 3, 453-472.

[DS14] S. Donaldson and S. Sun, Gromov-Hausdorff limits of Kahler manifolds and algebraic geometry, Acta Math. 213 (2014), no. 1, 63-106.

[DT92] W. Ding and G. Tian, Kähler-Einstein metrics and the generalized Futaki invariants, Invent. Math. 110, (1992), no. 2, 315-335.

[Fjt15a] K. Fujita, On K-stability and the volume functions of $\mathbb{Q}$-Fano varieties, arXiv:1508.04052 accepted by Proc. London Math. Soc.

[Fjt15b] K. Fujita, Optimal bounds for the volumes of Kähler-Einstein Fano manifolds, arXiv:1508.04578; accepted by Amer. J. Math.

[Fjt16a] K. Fujita, On Berman-Gibbs stability and K-stability of $\mathbb{Q}$-Fano varieties, Compos. Math. 152 (2016), no. 2, 288-298.

[Fjt16b] K. Fujita, A valuative criterion for uniform $K$-stability of $\mathbb{Q}$-Fano varieties, arXiv:1602.00901; accepted by J. Reine Angew. Math.

[FS90] A. Fujiki and G. Schumacher, The moduli space of extremal compact Kähler manifolds and generalized Weil-Petersson metrics, Publ. Res. Inst. Math. Sci. 26 (1990), no. 1, 101-183.

[Ham51] P. C. Hammer, The centroid of a convex body, Proc. Amer. Math. Soc. 2, (1951), 522-525.

[KKL12] A.-S. Kaloghiros, A. Küronya and V. Lazić, Finite generation and geography of models, arXiv:1202.1164 to appear in Advanced Studies in Pure Mathematics, Mathematical Society of Japan, Tokyo.

[KM98] J. Kollár and S. Mori, Birational geometry of algebraic varieties, With the collaboration of C. H. Clemens and A. Corti. Cambridge Tracts in Math., 134, Cambridge University Press, Cambridge, 1998.

[Li16] C. Li, K-semistability is equivariant volume minimization, arXiv:1512.07205 2 .

[Liu] Y. Liu, private communication.

[LM09] R. Lazarsfeld and M. Mustaţă, Convex bodies associated to linear series, Ann. Sci. Éc. Norm. Supér. 42 (2009), no. 5, 783-835.

[LWX14] C. Li, X. Wang and C. Xu, Degeneration of Fano Kähler-Einstein varieties, arXiv:1411.0761

[Mab08] T. Mabuchi, K-stability of constant scalar curvature, arXiv:0812.4903.

[Mab09] T. Mabuchi, A stronger concept of K-stability, arXiv:0910.4617.

[Od10] Y. Odaka, On the GIT stability of polarised varieties - a survey -, Proceeding of Kinosaki algebraic geometry symposium 2010.

[Od13] Y. Odaka, On the moduli of Kähler-Einstien Fano manifolds, Proceeding of Kinosaki algebraic geometry symposium 2013, available at arXiv:1211.4833. 
[Od15] Y. Odaka, Compact moduli spaces of Kähler-Einstein Fano varieties, Publ. Res. Inst. Math. Sci. 51 (2015), no. 3, 549-565.

[OS12] Y. Odaka and Y. Sano, Alpha invariants and $K$-stability of $\mathbb{Q}$-Fano varieties, Adv. Math. 229 (2012), no. 5, 2818-2834.

[OSS16] Y. Odaka, C. Spotti and S. Sun, Compact moduli spaces of Del Pezzo surfaces and Kähler-Einstein metrics, J. Differential Geom. 102 (2016), no. 1, $127-172$.

[OSY12] H. Ono, Y. Sano and N. Yotsutani, An example of an asymptotically Chow unstable manifold with constant scalar curvature, Ann. Inst. Fourier (Grenoble) 62 (2012), no. 4, 1265-1287.

[SSY14] C. Spotti, S. Sun and C. Yao, Existence and deformations of KählerEinstein metrics on smoothable $\mathbb{Q}$-Fano varieties, arXiv:1411.1725,

[Sto09] J. Stoppa, K-stability of constant scalar curvature Kähler manifolds, Adv. Math. 221 (2009), no. 4, 1397-1408.

[Tia87] G. Tian, On Kähler-Einstein metrics on certain Kähler manifolds with $C_{1}(M)>0$, Invent. Math. 89 (1987), no. 2, 225-246.

[Tia97] G. Tian, Kähler-Einstein metrics with positive scalar curvature, Invent. Math. 130 (1997), no. 1, 1-37.

[Tia15] G. Tian, K-stability and Kähler-Einstein metrics, Comm. Pure Appl. Math. 68 (2015), no. 7, 1085-1156; Corrigendum, 68 (2015), no. 11, 2082-2083.

Research Institute for Mathematical Sciences, Kyoto University, Kyoto 6068502. JAPAN

$$
\text { fujita@kurims.kyoto-u.ac.jp }
$$

Department of Mathematics, Kyoto University, Kyoto 606-8502. JAPAN

$$
\text { yodaka@math.kyoto-u.ac.jp }
$$

